\title{
Objects Tracking in Images Sequence using Local Binary Pattern (LBP)
}

\author{
H. Rami \\ LETS Laboratory, Physics \\ Department, Mohamed V \\ University, Faculty of Science, \\ Rabat, Morocco
}

\author{
M. Hamri \\ LETS Laboratory, Physics \\ Department, Mohamed V \\ University, Faculty of Science, \\ Rabat, Morocco
}

\author{
Lh. Masmoudi \\ LETS Laboratory, Physics \\ Department, Mohamed V \\ University, Faculty of Science, \\ Rabat, Morocco
}

\begin{abstract}
In this paper we present a method for objects tracking in images sequence. This approach is achieved into two main steps. In the first one, we constructed the Local Binary Pattern (LBP) histogram pattern of each image in the sequence and the reference pattern. In the second one, we perform the algorithm by the pattern selected based on a distance measures to find similarity between two histograms. The maximum LBP histogram distance gives best results than the chi-square one. The proposed approach has been tested on synthetic and real sequence images and the results are satisfactory.
\end{abstract}

\section{Keywords}

Sequence image, Computer vision, Tracking, LBP histogram, chi-square distance.

\section{INTRODUCTION}

Tracking systems is important in computer vision. It is applied in different domain, for example in video surveillance and human computer interfaces (HCI). Various methods can be found in the literature and can be roughly classified into two basic categories.

The first category is the algorithms that estimate the absolute positions of the pixel in each image independently. This category includes the center-of-mass, or centroid algorithm [1,2] and direct fits of Gaussian curves to the intensity profile $[3,4]$.

The second category includes algorithms that estimate the change in position of a pixel by comparing an image to one subsequent. This category includes cross-correlation method [5,6,7], and SAD algorithm method [8]. The use of the second category is well known and commonly used in tracking for sequence image. And it is commonly used in tracking vision for the visual matching problem [9].

But the SAD method suffers from the sensitivity to intensity scaling of the image and the template $[11,12,13]$. And the ZNCC method presents an ambiguity in the area with similar brightness or similar texture. It is demonstrated that the LBP (Local Binary Pattern) is mainly characterized by the invariance to monotonic changes in gray-scale and fast computation, and it has proven performance background in texture Classification [10]. While operating in gray-scale color space, LBP is also robust to illumination changes.

Texture, which has not enjoyed major attention in tracking applications, provides a good option to enhance the power of color descriptors. In this way we propose to use the LBP [10] histograms to tracking the motif in a sequence of images. In order to show the feasibility of the proposed method, it is tested and applied to both real image sequences and synthesized image sequences.

\section{LOCAL BINARY PATTERNS (LBP)}

The basic LBP features have performed very well in various applications, including texture classification and segmentation, image retrieval and surface inspection [10]. The local binary pattern (LBP) texture analysis operator is defined as a gray-scale invariant texture measure, derived from a general definition of texture in a local neighborhood. The local binary pattern (LBP) operator was first introduced as a complementary measure for local image contrast [15]. The figure 1 shows an example of LBP calculation. LBP is a powerful operator of texture description. It labels the pixels of an image region by thresholding the 3-by-3 neighborhood of each pixel with the center value and by arraying the result to binary codes $[10,15]$.

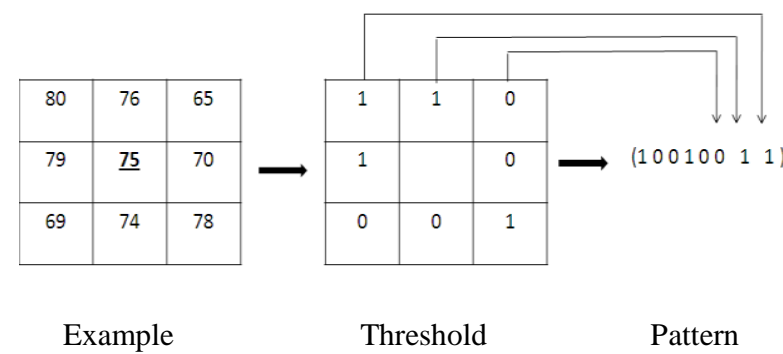

Figure 1: Example of LBP calculation: pattern $=10010011 ; \mathrm{LBP}=(10010011)_{2}=147$

The 256-bin histogram of the labels computed over an image can be used as a texture descriptor. Each bin of histogram (LBP code) can be regarded as a micro-texton. Local primitives which are codified by these bins include different types of curved edges, spots, flat areas, etc. Figure 3 shows some examples.[15,17] 


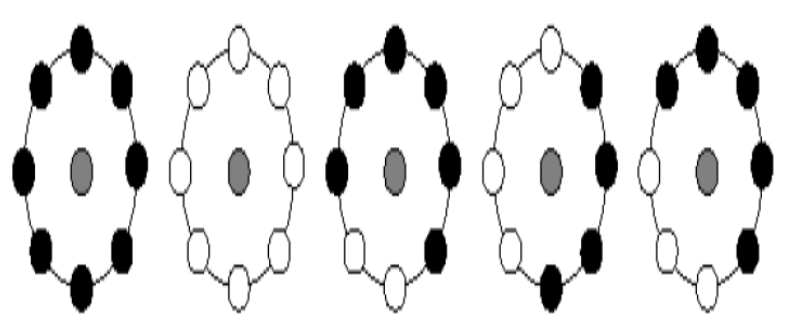

spot Spot/flat Line end Edge Corner Figure 2: Examples of texture primitives

Local binary pattern is a simple description operator of local texture. It can resist to the changes of illumination [10, 11]. And it has proven performance background in texture classification [10]. In recent years, the LBP operator has been used for texture classification, face recognition, image retrieval and other fields.

\section{HISTOGRAM DISTANCE}

The use bin-to-bin distances for comparing histograms is very important. This practice assumes that the histogram domains are aligned. This distance depends on the number of bins. If it is low, the distance is robust, but not discriminative, if it is high, the distance is discriminative, but not robust. Distances that take into account cross-bin relationships (crossbin distances) can be both robust and discriminative. There are two kinds of cross-bin distances. The first is the Quadratic-Form distance [19]. Let P and Q be two histograms and A the bin-similarity matrix. The Quadratic- Form distance is defined as:

$$
Q F^{A}(P, Q)=\sqrt{(P-Q)^{T} A(P-Q)}
$$

When the bin-similarity matrix $\mathrm{A}$ is the inverse of the covariance matrix, the Quadratic-Form distance is called the Mahalanobis distance. The second type of distance that takes into account cross-bin relationships is the Earth Mover's

Distance (EMD) [20].

In many natural histograms the difference between large bins is less important than the difference between small bins and should be reduced. The Chi-Squared (x2) is a histogram distance that takes this into account. It is defined as:

$$
\chi^{2}(P, Q)=\frac{1}{2} \sum_{i} \frac{\left(P_{i}-Q_{i}\right)^{2}}{\left(P_{i}+Q_{i}\right)}
$$

The $\boldsymbol{X}_{2}$ histogram distance comes from the $\mathrm{x} 2$ test-statistic

[21] where it is used to test the fit between a distribution and observed frequencies. Chi-square histogram distance is one of the distance measures that can be used to find dissimilarity between two histograms. $\mathrm{x} 2$ was successfully used for texture and object categories classification [22, 23, 24], near duplicate image identification[25], local descriptors matching [26], shape classification [27, 28] and boundary detection [29].

\section{PROPOSED METHOD}

The proposed method is achieved in two main steps. In the first one, we constructed the Local Binary Pattern (LBP) histogram pattern of each image in the sequence and the reference pattern. In the second one, we perform the algorithm by the pattern selected based on a distance measures to find similarity between two histograms. The flowing we present the algorithm used in this stud.

\section{Algorithm:}

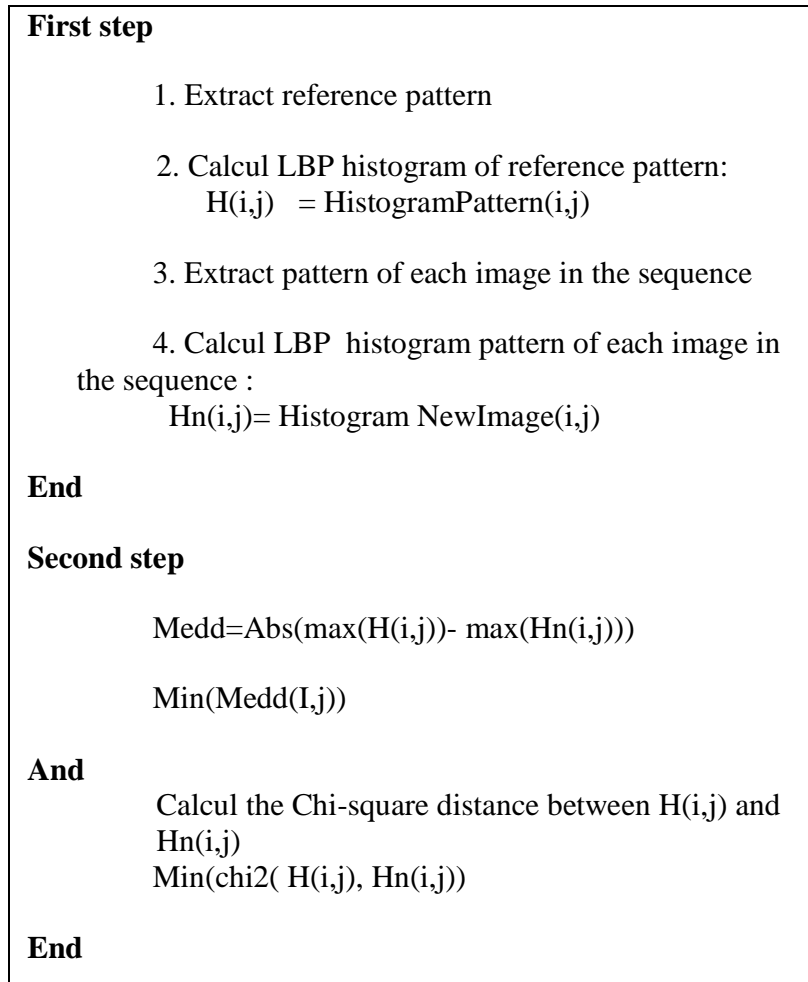

\section{EXPERIMENTAL RESULT}

In this section, we present the experimentation results of into tracking image sequences. Real image sequences and synthesized image sequences are considered. For evaluation the algorithm tracking results we use Euclidean distance.

\subsection{Synthesized sequence image}

We used a sequence of grayscale image containing a moving ball. This database gives returns to Strauss [18]. Table 1 shows the cumulative Euclidean distance $r$ from the pixel 
position for each images $i$ and $(i+1)$ for the similarity measure using chi-square and maximum histogram.

\begin{tabular}{|c|c|c|c|c|c|c|c|c|c|c|c|c|c|c|c|c|c|c|c|}
\hline sequence $\mathrm{N}^{\circ}$ & 1 & 2 & 3 & 4 & 5 & 6 & 7 & 8 & 9 & 10 & 11 & 12 & 13 & 14 & 15 & 16 & 17 & 18 & 19 \\
\hline Chi-square & 16 & 21 & 66 & 86 & 91 & 164 & 232 & 261 & 278 & 312 & 337 & 366 & 418 & 455 & 472 & 509 & 554 & 590 & 616 \\
\hline Max-histogram & 10 & 26 & 51 & 52 & 68 & 136 & 156 & 181 & 190 & 239 & 275 & 300 & 336 & 361 & 386 & 426 & 466 & 502 & 527 \\
\hline
\end{tabular}

Table 1: Cumulative Euclidean distance for the two method

Figure 3 presents the evolution of the position of pixels for each image using de cumulative Euclidean distance. The figure shows that the similarity measure uses the maximum histogram is below the chi-square. Therefore, the maximum LBP histogram distance gives best results than the chi-square one. Finally, we present examples of image sequence using the max histogram. From the results, it can be seen that its performance is acceptable for the synthetic sequence images.

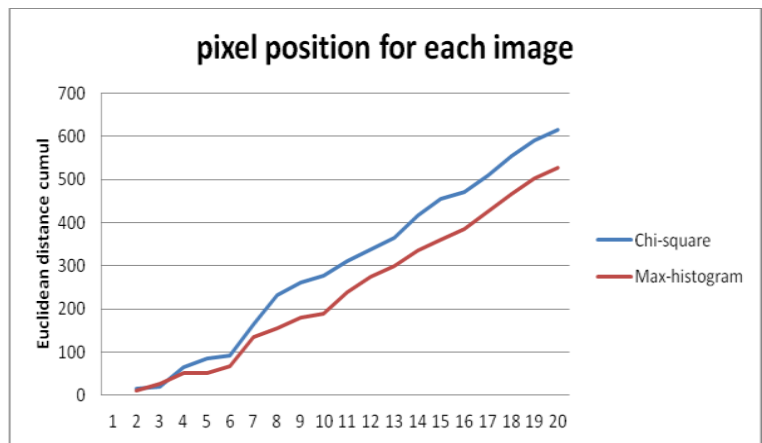

Figure 3 :evolution of the position of pixels for each image using de cumulative Euclidean distance
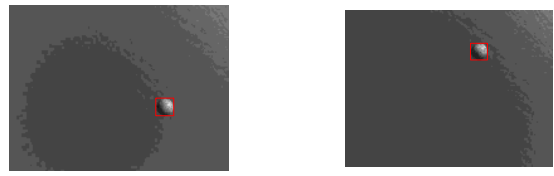

Image45

Image 60

Figure 4 : same image using max-histogram for tracking the ball

\subsection{Real sequence image}

In order to compare the performances of the method we have considered a real sequence image. We have used the video realized by Sargi [30] for tracking the face. Table 2 present the cumulative Euclidean distance from the pixel position for each images $i$ and $(i+1)$ for the similarity measure using chisquare and maximum histogram. The table shows that the values relate to the measurement of Chi-square augment rapidly. In particular for image 6 and 7 the value of distance changes from 156 to 253, per against the distance for Maxhistogram increases in a progressive manner.

Figure 5 presents the evolution of the position of pixels for each image using de cumulative Euclidean distance. The figure shows that the similarity measure uses the maximum histogram is below the chi-square. Therefore, the maximum LBP histogram distance gives best results than the chi-square one. Finally, we present examples of image sequence using the max histogram. From the results, it can be seen that its performance is acceptable for the synthetic sequence images. 


\begin{tabular}{|c|c|c|c|c|c|c|c|c|c|c|c|c|c|c|c|c|}
\hline Image Sequence $\mathrm{N}^{\circ}$ & 1 & 2 & 3 & 4 & 5 & 6 & 7 & 8 & 9 & 10 & 11 & 12 & 13 & 14 & 15 & 16 \\
\hline Chi-square & 16 & 21 & 30 & 47 & 51 & 164 & 253 & 303 & 319 & 348 & 373 & 402 & 438 & 478 & 494 & 534 \\
\hline Max-histogram & 0 & 128 & 165 & 190 & 190 & 255 & 255 & 272 & 272 & 282 & 282 & 291 & 291 & 344 & 344 & 426 \\
\hline
\end{tabular}

Table 2: Cumulative Euclidean distance for the two method

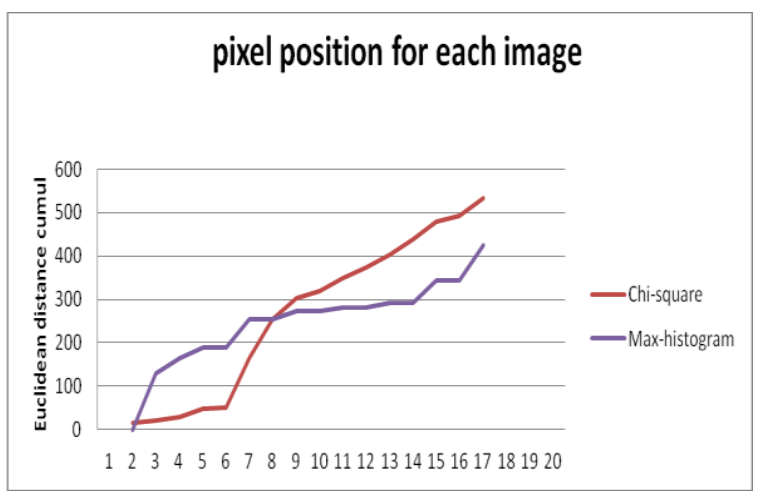

Figure 5 :evolution of the position of pixels for each image using de cumulative Euclidean distance

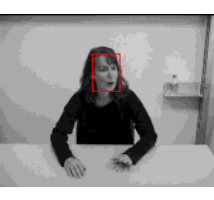

Image 1

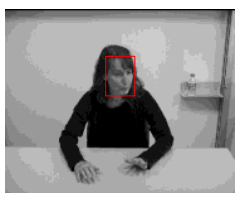

Image 5

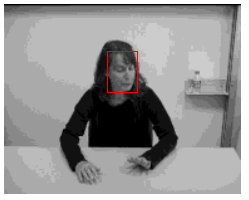

Image 10

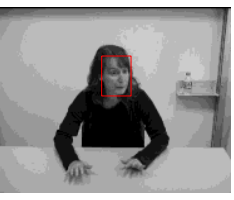

Image 15

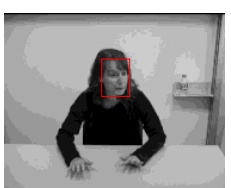

Image 17

Figure : same image using max-histogram for tracking the face

\section{CONCLUSION}

In this paper a method for objects tracking in images sequence using Local Binary Pattern (LBP). For evaluation the algorithm tracking results we use the cumulative Euclidean distance from the pixel position for each images. The maximum LBP histogram distance gives best results than the chi-square one. From the results, it can be seen that its performance is acceptable for both the synthetic and real sequence images. In the future work, we will exploit the information color for constructed the LBP operator.

\section{REFERENCES}

[1] Ghosh and Webb. 1994. Automated detection and tracking of individual and clustered cell surface low density lipoprotein receptor molecules.Biophys. J.66:1301-1318.
[2] Lee, and al. 1991. Direct observation of Brownian motion of lipids in a membrane.Proc. Nat. Acad. Sci. U.S.A.88:6274 - 6278 .

[3] Anderson, and al. 1992. Tracking of cell surface receptors by fluorescence digital imaging microscopy using a charge-coupled device cam-era.J. Cell Sci.101:415- 425.Tavel, P. 2007 Modeling and Simulation Design. AK Peters Ltd.

[4] Smith, and al. 1999. A direct comparison of selectinmediated transient, adhesive events using high temporal resolution.Biophys. J.77:3371-3383.

[5] Gelles, and al.1988. Tracking kinesin-driven movements with nanometre-scale precision.Nature.331:450 - 453.

[6] Kusumi, and al. 1993. Confined later diffusion of membrane receptors as studied by single particle tracking (nanovid microscopy). Effects of calcium-induced differentiation in cultured endothelial cells. Biophys. J.65:2021-2040. 
[7] ] Guilford and Gore, 1995. The mechanics of arteriole interstitium interaction. Microvas. Res.50:260 -287.

[8] Vanne, and al, 2006. "A High-Performance Sum of Absolute Difference Implementation for Motion Estimation," Circuits and Systems for Video Technology, IEEE Transactions on, vol.16, no.7, pp.876-883.

[9] Kanade and Okutomi.1994. A stereo matching algorithm with an adaptive window: theory and experiment. IEEE Transactions for Pattern Analysis and Machine Intelligence 16, 920-932.

[10] Ojala and al, 2002. Multiresolution gray-scale and rotation invariant texture classification with local binary patterns. IEEE Transactions on Pattern Analysis and Machine Intelligence, 24(7): 971-987

[11] Cho, and Yun, 2005 .Selective-attention correlation measure for precision video tracking. IEICE Trans. Inf. Syst. E88-D(5), 1041-1049

[12] Bohs, and al.1999. Speckle tracking for multidimensional flow estimation. Ultrasonics 38, 369-375 (2000)

[13] Heikkil and Pietik ,2006. A texture-based method for modeling the background and detecting moving objects. IEEE Transactions on Pattern Analysis and Machine Intelligence, 28:657-662

[14] Timo Ahonen and al, 2009. Rotation invariant image description with local binary pattern histogram

[15] Ojala T, Pietikäinen M \& Harwood D (1996) A comparative study of texture measures with classification based on featured distribution. Pattern Recognition, 29(1):51-59.

[16] A. Hadid, M. Pietikainen and T. Ahonen. A Discriminative Feature Space for Detecting and Recognizing Faces. Proc of CVPR 2004.

[17] Jo Chang-yeon, "Face Detection using LBP features," Final Project Report, December 2008.

[18] Olivier STRAUSS Laboratoire d'Informatique, de Robotique et de Micro-électronique de Montpellier

[19] Département Robotique LIRMM 161, Rue ADA 34392 Montpellier CEDEX 5 France
[20] Hafner, J., Sawhney, H., Equitz, W., Flickner, M., Niblack, W.: Efficient color histogram indexing for quadratic form distance functions. PAMI (1995)

[21] Rubner, Y., Tomasi, C., Guibas, L.J.: The earth mover's distance as a metric for image retrieval. IJCV (2000)

[22] Snedecor, G., Cochran, W.: Statistical Methods, ed 6. Ames, Iowa (1967)

[23] Cula, O., Dana, K.: 3D texture recognition using bidirectional feature histograms. IJCV (2004)

[24] Zhang, J., Marszalek, M., Lazebnik, S., Schmid, C.: Local features and kernels for classification of texture and object categories: A comprehensive study. IJCV (2007) 3

[25] Varma,M., Zisserman, A.: A statistical approach to material classification using image patch exemplars. PAMI (2009) 3

[26] Xu, D., Cham, T., Yan, S., Duan, L., Chang, S.: Near Duplicate Identification with Spatially Aligned Pyramid Matching. CSVT (accepted) 3

[27] Forss'en, P., Lowe, D.: Shape Descriptors for Maximally Stable Extremal Regions. In: ICCV.(2007) 3

[28] Belongie, S., Malik, J., Puzicha, J.: Shape matching and object recognition using shape contexts. PAMI (2002) 3 , 11

[29] Ling, H., Jacobs, D.: Shape classification using the inner-distance. PAMI (2007) 3, 11

[30] Martin, D., Fowlkes, C., Malik, J.: Learning to detect natural image boundaries using local brightness, color, and texture cues. PAMI (2004).

[31] Mehmet Emre Sargin and al.2005. Combined GestureSpeech Analysis and Synthesis eNTERFACE05 Workshop in Mons, Belgium. 